\title{
Single-grain results from an EMCCD-based imaging system
}

Thomsen, Kristina Jørkov; Kook, Myung Ho; Murray, Andrew; Jain, Mayank; Lapp, Torben

\section{Published in:}

Radiation Measurements

Link to article, DOI:

10.1016/j.radmeas.2015.02.015

Publication date:

2015

Document Version

Peer reviewed version

Link back to DTU Orbit

Citation (APA):

Thomsen, K. J., Kook, M. H., Murray, A., Jain, M., \& Lapp, T. (2015). Single-grain results from an EMCCDbased imaging system. Radiation Measurements, 81, 185-191. https://doi.org/10.1016/j.radmeas.2015.02.015

\section{General rights}

Copyright and moral rights for the publications made accessible in the public portal are retained by the authors and/or other copyright owners and it is a condition of accessing publications that users recognise and abide by the legal requirements associated with these rights.

- Users may download and print one copy of any publication from the public portal for the purpose of private study or research.

- You may not further distribute the material or use it for any profit-making activity or commercial gain

- You may freely distribute the URL identifying the publication in the public portal

If you believe that this document breaches copyright please contact us providing details, and we will remove access to the work immediately and investigate your claim. 


\title{
Single-grain results from an EMCCD-based imaging system
}

Thomsen, K.J. ${ }^{1}{ }^{*}$, Kook, M.H. ${ }^{1}$, Murray, A.S. ${ }^{2}$, Jain, M. ${ }^{1}$, Lapp, $\mathrm{T}^{1}$.

${ }^{1}$ Center for Nuclear Technologies, Technical University of Denmark, DTU Ris $\varnothing$ Campus, DK-4000 Roskilde, Denmark

${ }^{2}$ Nordic Laboratory for Luminescence Dating, Department of Geoscience, Aarhus University, DTU Nutech, Risø Campus, DK-4000 Roskilde, Denmark

* Corresponding author: krth@dtu.dk

Key words: EMCCD imaging; OSL; TL; Quartz; Single-grain

\begin{abstract}
Here we compare the performance of an EMCCD-based imaging system with the standard laser-based single-grain Ris $\varnothing$ attachment. We first compare gamma dose distributions and the relative sensitivity of the two instruments is investigated using a single sample, by comparing the number of grains accepted into a dose distribution. EMCCD cross-talk is shown to be of concern at low light levels. We also make use of the fact that the EMCCD can observe TL signals from individual grains to examine the use of the correlation between the quartz $110^{\circ} \mathrm{C}$ TL peak and the fast component OSL signal to correct for sensitivity change. Finally, we present the OSL dose distributions from a set of both well-bleached and poorly-bleached sedimentary samples. From a comparison of the measured doses, we conclude that the two instruments give indistinguishable dose estimates and dispersions, despite the fact that the laser-based system is effectively about four times as sensitive as the EMCCD.
\end{abstract}

\section{INTRODUCTION}

Photomultiplier tubes are used almost exclusively in the measurement of luminescence signals, especially in luminescence dating, because of their sensitivity and ease of use. They are particularly sensitive in the blue and near UV part of the spectrum, they have a large dynamic range and a low dark count rate, but they are integral counting devices - conventionally they do not provide spatial information. In dating applications, this limits their usefulness to measurement of a single sample, whether a multi-grain aliquot, or a single grain. Any information on the original context of the grain(s) is necessarily lost during chemical separation and treatment. An imaging detector, on the other hand, would permit the mapping of 
luminescence from consolidated samples or slices. Even for prepared samples, imaging could allow the measurement of many individual grains simultaneously. It might even be possible to measure single grains without the need for mineral separation. Progress has been made in the use of sensitive charge coupled devices (CCD) to image TL (Spooner, 2000; Olko et al., 2008), IRSL (Duller et al., 1997, Greilich et al., 2002; Baril, 2004; Greilich, 2004; Greilich and Wagner, 2006) and OSL (Clark-Balzan and Schwenninger, 2012; Richter et al., 2013; Mundupuzhakal et al., 2014), but although some demonstrated that a CCD-based system can be sufficiently sensitive (especially the most recent publications) none of these studies were able to develop the technology to a point where it could be used for routine analysis. In contrast, Kook et al., (these proceedings) have recently used an example of the latest generation of such devices (electron multiplier coupled CCD, or EMCCD) in a new attachment to the Ris $\varnothing \mathrm{TL} / O S L$ reader, in which they use software to automatically locate individual grains during repeated analysis. This allows, for the first time, completely automatic image analysis of single grain luminescence signals, and so offers an alternative to the well-established laser-based single grain attachment to the Ris $\varnothing$ reader (Bøtter-Jensen et al., 2003).

Here we test the application of this EMCCD-based imagining system and compare the results with those obtained using the laser-based attachment. Dose distributions measured using gamma-irradiated sensitised quartz are considered first, and then the sensitivities of the two measurement systems are compared. The degree of spill-over of light from one grain to another (cross-talk) is evaluated. Stimulation curve decay rates are known to vary considerably in the laser-based system; we use the EMCCD system to help determine whether this variability is instrumental, or is inherent in the grains. The imaging system makes the measurement of TL signals from individual grains practical for the first time, and this new possibility is used to test whether the $110^{\circ} \mathrm{C}$ TL peak has potential as a monitor of sensitivity change in individual grains. Finally measurements of natural single-grain dose distributions obtained using the two systems are compared.

\section{EXPERIMENTAL DETAILS}

All experiments used automated TL/OSL Ris $\varnothing$ DA-20 readers (Bøtter-Jensen et al., 2010) equipped with calibrated ${ }^{90} \mathrm{Sr} /{ }^{90} \mathrm{Y}$ beta sources as the measurement platform. In one measurement setup (EMCCDsystem) optical stimulation is achieved using the standard Ris $\varnothing$ stimulation head containing arrays of blue $(470 \pm 30 \mathrm{~nm})$ and infrared (IR, $870 \pm 40 \mathrm{~nm})$ stimulation LEDs, providing stimulation powers at the sample position of approximately 40 and $130 \mathrm{~mW} / \mathrm{cm}^{2}$, respectively. In this setup all individual grains are stimulated simultaneously. The detection of both TL and OSL signals from quartz used an EMCCD detector (Kook et al., these proceedings) through $6 \mathrm{~mm}$ of a coated Hoya U-340 glass filter. 
In a second setup (XY system) optical stimulation was achieved using the Ris $\varnothing$ single grain laser attachment (Bøtter-Jensen et al., 2003) employing a $10 \mathrm{~mW} \mathrm{Nd:YVO}{ }_{4}$ solid-state diode-pumped laser emitting at $532 \mathrm{~nm}$. The laser is focused ( $20 \mu \mathrm{m}$ spot size) sequentially onto a square grid of grain holes in a sample disc. Duller et al. (1999) stated that the maximum power density at the sample is $\sim 50 \mathrm{~W} / \mathrm{cm}^{2}$ but in fact it is probably closer to $14 \mathrm{~W} / \mathrm{cm}^{2}$ (assuming that the laser deposits its energy in a circle with a diameter of $300 \mu \mathrm{m}$, Thomsen et al., submitted). An EMI 9635QB photomultiplier in combination with 7.5 mm Hoya U-340 filters is used to measure the OSL signals.

In a third setup, a new automated TL/OSL Detection and Stimulation Head (DASH, see Lapp et al., these proceedings) is used with the EMCCD; this head incorporates blue $\left(470 \mathrm{~nm}, \sim 100 \mathrm{~mW} / \mathrm{cm}^{2}\right)$, green $\left(530 \mathrm{~nm}, \sim 50 \mathrm{~mW} / \mathrm{cm}^{2}\right)$ and IR $\left(870 \mathrm{~nm}, \sim 130 \mathrm{~mW} / \mathrm{cm}^{2}\right)$ LEDs for optical stimulation. This new head also includes two automated filter changers enabling the use of different filter combinations in the same measurement sequence; various combinations of UV/blue detection filters are used in this work.

\subsection{SAMPLE PRESENTATION}

All single-grain measurements reported here have been obtained by loading individual sand-sized grains into aluminium sample discs containing a 10 by 10 array of grain holes (depth and diameter of 200 or 300 $\mu \mathrm{m}$ ) spaced on a $600 \mu \mathrm{m}$ square grid (Duller et al., 1999) for three main reasons: i) it is a convenient way of comparing the OSL measured from individual grains using the PMT (and laser stimulation) and the EMCCD (LED stimulation), ii) the inevitable cross-talk in the EMCCD-imaging system is reproducible and quantifiable, iii) the exact position of individual grains from measurement to measurement is easily determined using the single-grain disc's identification holes, and thus the risk of additional scatter in the measured dose distributions due to misidentification of individual grains is minimised. In practice, we collect an optical image of the sample disc after each luminescence measurement, by illuminating with a very low power infrared (IR) diode. This image is subsequently software analysed to determine the exact position of the individual grains. Using this position information, the luminescence image files are analysed using preselected regions of interest (ROI) and the light sums from these ROI automatically converted into standard BINX files, which can be analysed conventionally (see Kook et al., these proceedings, for details).

\section{RESULTS}

\subsection{GAMMA DOSE DISTRIBUTION}

In Figure 1 we show examples of single-grain dose response curves (DRC) and single-grain stimulation curves from the XY system (Figure 1a) and the DASH system (EMCCD; Figure 1b) obtained using a heated (sensitised) and gamma dosed (4.81 $\pm 0.10 \mathrm{~Gy}$ ) quartz sample (calibration quartz Batch 90; Hansen et al., 
these proceedings). The sample was measured using a test dose of $4 \mathrm{~Gy}$ and standard preheat and cutheat temperatures of $260^{\circ} \mathrm{C}$ for $10 \mathrm{~s}$ and $220^{\circ} \mathrm{C}$. In Figure $1 \mathrm{c}, \mathrm{d}$ we show resulting dose distributions, each measured using 2 single-grain discs loaded with calibration quartz. In the XY system (Figure 1c), 193 grains were accepted (uncertainty on the natural test dose response $\mathrm{s}_{\mathrm{TN}}<30 \%$ ), the average test dose response is $2000 \pm 200$ counts summed over $60 \mathrm{~ms}$, the relative standard deviation of the dose distribution is $14 \%$, and the relative over-dispersion (OD) is $10.4 \pm 0.7 \%$ (assuming Poisson statistics). In the DASH system (Figure $1 \mathrm{~d}$ ), the corresponding numbers are: 198 grains, $960 \pm 100$ counts summed over $0.5 \mathrm{~s}, 13 \%$, and OD of $8.5 \pm 0.7 \%$. (Note the different summation times reflect the different stimulation rates.) Unfortunately, one cannot simply assume Poisson statistics for the EMCCD data; the EMCCD itself introduces additional uncertainty which must be taken into account. Kook et al. (these proceedings) show that EMCCD raw data can be converted into Poisson distributed data by dividing by 2 . Then the variance is correctly estimated by the count (as is usually assumed for PM tube data). In this case, such treatment reduces the apparent OD to $7.1 \pm 0.8 \%$. Nevertheless, the average test dose response from the $X Y$ system is about twice that from the EMCCD; when converted to Poisson statistics, this factor increases to 4 . It may be that this difference in OD can be attributed to the additional uncertainties induced by steering the laser. This contribution does not exist in the EMCCD system.

\subsection{SENSITIVITY}

Previous attempts to use imaging-based techniques for quartz at a single-grain level have been restricted by detector sensitivity. In this section we compare the sensitivity of the EMCCD camera to that of the PMT. A previously measured disc of calibration quartz was given a dose of $50 \mathrm{~Gy}$, preheated to $240{ }^{\circ} \mathrm{C}$ for $10 \mathrm{~s}$ and stimulated with blue LEDs (standard stimulation head) for $40 \mathrm{~s}$ at $125^{\circ} \mathrm{C}$. The emitted OSL was detected using the EMCCD camera. The disc was then given a dose of $50 \mathrm{~Gy}$ and preheated to $240{ }^{\circ} \mathrm{C}$ for 10 $s$ before being transferred (in darkness) to the XY system, where each grain was measured sequentially using the green laser for $1.9 \mathrm{~s}$ at $125^{\circ} \mathrm{C}$. The rate of decay observed using the blue LEDs is 10 times slower than that observed using the green laser (see insets in Figure 1a,b) because of the difference in optical cross-section as well as stimulation power. To ensure that the same signal is being compared and thus get a sensible estimate of the sensitivity of the EMCCD-setup compared to the conventional single-grain laser setup we sum the entire OSL signals (minus a background). In Figure 2 we show these signals obtained using the EMCCD as a function of those obtained using the single-grain laser attachment, together with a linear fit to the data - the sensitivity of EMCCD system is about $50 \%$ of that of the PMT system. However, at low signal intensities there are several grains for which the signal is significantly higher for the EMCCD than for the PMT (see inset to Figure 2). This results from cross-talk, and is considered in the next section. 


\subsection{CROSS-TALK}

In the $\mathrm{XY}$ system individual grains are loaded into special single-grain discs, where they are kept in a fixed geometry. They are subsequently stimulated sequentially by a tightly focused laser. Duller (2012a) measured the optical cross-talk of such systems to be on average $~ 0.04 \%$ for immediately adjacent grains, i.e. while stimulating a grain in the measurement position the signal in an adjacent unmeasured grain may decrease by $0.04 \%$. However, the stimulation power density to adjacent grains is so low that it does not give rise to detectable OSL decay in a typical stimulation time of 1 to 2 seconds (Duller, 2012a) and thus the contribution from such optical cross-talk will be removed during standard background subtraction. The effect on the adjacent grain remains, but this cancels out in SAR measurements, because every measurement is preceded by the same cross-talk effect. In the EMCCD-based imaging system cross-talk is different; all grains are stimulated simultaneously (using LEDs) and the amount of optical cross-talk is expressed as the amount of spill-over of light from one grain into the integration ROI of an adjacent grain. This will depend both on the distance to the nearest grain and the diameter of the ROI. One of the major reasons for mounting grains in single-grain discs for measurement in the EMCCD system is to ensure that optical cross-talk is both constrained and reproducible.

Figure 3a shows an EMCCD OSL image of a single-grain disc loaded with calibration quartz; this sample had been given a dose of $50 \mathrm{~Gy}$ and preheated to $240^{\circ} \mathrm{C}$ for $10 \mathrm{~s}$ before stimulation. This image is of the same measurement as is shown in Figure 2, and the grain holes marked with red circles provided the red data points in that figure. All the grain positions giving the red data are immediately adjacent to very bright grains. Figure $3 b$ shows the blue LED OSL stimulation curve (EMCCD detection) for such a bright grain (G82) and Figure $3 c$ shows the stimulation curve obtained using green laser stimulation (PMT detection) for the same grain. The insets show the corresponding stimulation curves for G81, immediately adjacent to the bright grain G82. In the laser-based system no detectable decay is observed for G81 whereas the EMCCD data show a clear fast decaying signal due to an optical cross-talk of $\sim 1 \%$, predominantly from the bright grain in position 82 . Kook et al. (these proceedings) have made a detailed study of this problem, and estimates that the average cross-talk using the integration conditions employed here (ROI of $450 \mu \mathrm{m}$ in diameter) and conclude that the on average about $0.8 \%$ of the light from one grain will be included in the adjacent integration intervals. This cross-talk could be reduced by decreasing the diameter of the ROIs, but this would, of course, be at the expense of reduced sensitivity.

\subsection{VARIABILITY IN SINGLE GRAIN DECAY RATES}

In quartz OSL dating the so-called fast component of the OSL signal (e.g. Jain et al., 2003; Singarayer and Bailey, 2003) is the preferred dating signal because of its ease of bleaching and stability. If other 
components contribute significantly to the OSL signals inaccurate dose estimates may result (e.g. Bailey, 2003; Choi et al., 2003). Figure 4a shows six blue stimulation curves measured at a power density of $\sim 25$ $\mathrm{mW} / \mathrm{cm}^{2}$ using $3 \mathrm{~mm}$ aliquots $(180-250 \mu \mathrm{m})$ of calibration quartz after a preheat of $260^{\circ} \mathrm{C}$ for $10 \mathrm{~s}$. The individual curves have been normalised and fitted using two decaying exponentials and a background. There is little variability between the individual curves and the initial signal is clearly dominated by the fast component. However when we measure this material using the laser-based single-grain attachment we observe a large variability in decay shapes between grains (see Figure $4 b$ ). Such variability in single-grain data is often attributed to different relative contributions of different components in different grains (e.g Ballerini et al., 2007; Duller, 2012b), although it has also been suggested that these observations may arise because of variations in effective stimulation power; this variation is expected to arise because of varying reflection of the tightly focussed laser light as it hits the surfaces of different grains (Thomsen et al., 2012; Thomsen et al., submitted). To investigate this hypothesis we have measured the same grains (calibration quartz) both using the single-grain green laser attachment (PMT detection) at both 90 and $10 \%$ and using a DASH equipped with blue and green LEDs (EMCCD detection). To quantify the rate of decay of individual grains we use the time it takes for the intensity to drop to $50 \%$ of its initial value as a proxy for the characteristic life time. The frequency distributions of these decay rates are shown in Figure 5 . The distributions for blue and green LED stimulation are approximately normal with average $T_{1 / 2}$ values of $0.27 \pm 0.01 s(n=89)$ and $6.1 \pm 0.1 s(n=89)$, respectively. The corresponding relative standard deviations are 23 and $22 \%$, respectively. However, the distributions for laser stimulation are positively skewed with modes of $\sim 0.015$ and $\sim 0.15 \mathrm{~s}$, for 90 and $10 \%$ stimulation power, respectively. The corresponding relative standard deviations are 53 and $57 \%$. These results confirm for the first time that the large variability in single-grain decay rates obtained using laser stimulation is mainly caused by differences in effective stimulation power between grains. Unfortunately, this makes OSL component separation in the analysis of data collected using the laser-based single-grain attachment of limited value.

\subsection{VARIABILITY IN DOSE RESPONSE CURVES}

It has been shown that green light stimulation may produce a better separation of the fast and the medium components in quartz OSL (Singarayer and Bailey, 2004; Thomsen et al., 2006) than blue light stimulation and thus one might expect to observe significantly different dose response curve (DRC) shapes using different stimulation wavelengths. We have measured calibration quartz SAR DRCs (Murray and Wintle, 2000; test dose of $20 \mathrm{~Gy}, \mathrm{PH}$ of $260^{\circ} \mathrm{C}$ for $10 \mathrm{~s}, \mathrm{CH}$ of $220^{\circ} \mathrm{C}$ and a high temperature blue bleach at $280^{\circ} \mathrm{C}$ for $40 \mathrm{~s}$ between SAR cycles) up to $256 \mathrm{~Gy}$ using four different stimulation modes: i) green laser stimulation

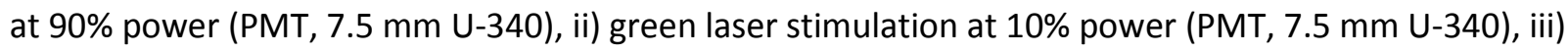
green LED stimulation (EMCCD, $3 \mathrm{~mm}$ U-340 and $1 \mathrm{~mm}$ coated U-340) and iv) blue LED stimulation 
(EMCCD, $5 \mathrm{~mm} \mathrm{U}-340$ and $1 \mathrm{~mm}$ coated U-340). First i) and ii) were measured by alternating from one SAR cycle to the next between the two power settings, and subsequently iii) and iv) were measured, this time alternating between green and blue stimulation, from one SAR cycle to the next. All DRCs were then fitted in Analyst (Duller, 2007) using a saturating exponential function passing through the origin, i.e. $L_{x} / T_{x}=I_{0} \times(1$ $-\exp \left(-D / D_{0}\right)$, where $I_{0}$ is the saturation value and $D_{0}$ is a constant describing the curvature of the $D R C$. The initial summation interval is $60 \mathrm{~ms}, 0.54 \mathrm{~s}, 7 \mathrm{~s}$ and $0.5 \mathrm{~s}$ for the four stimulation modes, respectively. In some grains we observe a clear difference in the DRCs depending both on stimulation power and energy, whereas in other grains this difference is less pronounced (data not shown). To summarize the results we have calculated average $D_{0}$ values for the same grains $(n=54)$ for all four stimulation modes, to give $54 \pm 5$

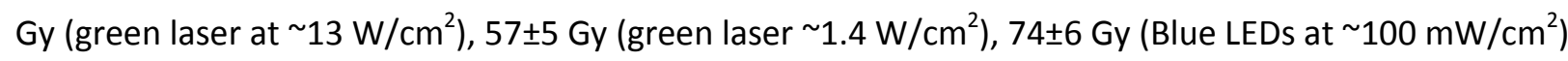
and $78 \pm 7 \mathrm{~Gy}$ (Green LEDs at $\sim 50 \mathrm{~mW} / \mathrm{cm}^{2}$ ). Thus, it would appear that, on average, we see a significant difference in $D_{0}$ values between LEDs and lasers, but not between blue and green LEDs; this difference presumably arises because of the large differences in stimulation power at the sample position, rather than because of differences in stimulation wavelength.

\subsection{TL $110^{\circ} \mathrm{C}$ PEAK SENSITIVITY CORRECTION}

One of the potential advantages of using imaging to obtain single-grain data is the possibility of measuring single grain thermoluminescence (TL). Murray and Roberts (1998) showed that sensitivity change of the $110^{\circ} \mathrm{C}$ TL quartz peak correlates with the OSL signal on a multi-grain level (although there was often a significant intercept in the relationship) and Murray and Wintle (2000) demonstrated that, for samples for which this intercept was small, the $110^{\circ} \mathrm{C} \mathrm{TL}$ intensity could be used to track sensitivity change even before making an OSL measurement. Singhvi et al. (2011) attempted to put this idea into practical effect by using the ratio of two $110^{\circ} \mathrm{C}$ TL peaks, one measured before and one after, the natural OSL measurement. They then used these measurements to correct for any sensitivity changes taking place during preheating and optical stimulation of the natural signal. An imaging detector offers the possibility of extending this correction to single-grain dose determinations; the majority of single-grain studies report unexplained scatter (both extrinsic and extrinsic) and it is conceivable that much of this scatter could be derived from first cycle sensitivity change. To test this concept, we measured a disc of calibration quartz (gamma dose of $4.81 \pm 0.10 \mathrm{~Gy}$ ) using a SAR protocol (total test dose of $4 \mathrm{~Gy}$, preheat of $260^{\circ} \mathrm{C}$ for $10 \mathrm{~s}$ and $\mathrm{CH}$ of $220^{\circ} \mathrm{C}$ ) but with the addition of a small test dose $(0.5 \mathrm{~Gy})$ and a $\mathrm{TL}$ to $180^{\circ} \mathrm{C}\left(\mathrm{TL}_{1}\right)$ before the preheat of the natural and regenerated OSL signals $\left(\mathrm{OSL}_{1}\right)$. This small test dose and $\mathrm{TL}$ to $180^{\circ} \mathrm{C}\left(\mathrm{TL}_{2}\right)$ was also inserted after the readout of the natural/regenerated OSL signal but before the measurement of the OSL response to the test 
dose $\left(\mathrm{OSL}_{2}\right)$. All signals were detected through $6 \mathrm{~mm}$ of Hoya U-340 (1 mm with coating) using the EMCCD system.

In Figure 6a we show the relationship between $\mathrm{TL}_{2}$ and $\mathrm{OSL}_{2}$ for six single grains. In general, we found a linear correlation passing through the origin between these two signals (although the correlation was questionable for some grains, e.g. see the two brightest grains in Figure 6a), giving us the possibility of correcting for first cycle sensitivity change on a single-grain level and possibly eliminate the unexplained scatter.

We analyse these data in three different ways: (i) using the standard OSL test dose correction, i.e. $\mathrm{OSL}_{1} / \mathrm{OSL}_{2}$. This gives an approximate normal dose distribution with a relative standard deviation of $14 \%$, $\mathrm{n}=99(\mathrm{OD}=8.8 \pm 1.0 \%)$, and (ii) correcting $\mathrm{OSL}_{1}$ by dividing by the preceding $\mathrm{TL}_{1}$ (similar to one of the approach suggested by Murray and Roberts, 1998). This also gives an approximately normal distribution but with a relative standard deviation of $22 \%, n=40$, and (iii) correcting $O S L_{1}$ by first dividing by the ratio of the two bracketing TLs, and then dividing by the OSL test dose response, i.e. $O S L_{1} \times\left(\mathrm{TL}_{2} / \mathrm{TL}_{1}\right) / \mathrm{OSL}_{2}$. In contrast to (i) and (ii) this correction is only applied to the natural cycle (Singhvi et al., 2011). This also gives an approximately normal dose distribution with a relative standard deviation of $25 \%, n=40$. The resulting dose distributions are shown as histograms in Figure $6 b, c, d$. Thus, for this sample at least it is not possible to reduce the observed scatter by using the TL response to a test dose before the measurement of $L_{x}$. It is interesting to note that for this sample the average ratio between $\mathrm{TL}_{2}$ and $\mathrm{TL}_{1}$ is $\sim 85 \%$ very similar to the values reported by Murray and Wintle (2000) for large aliquots of a heated and an unheated sample. However, we have not yet tested this approach on natural samples.

\subsection{DOSE DISTRIBUTIONS}

All the above discussion has been based on signals from calibration quartz, which is unusually sensitive. We now turn to measurements using quartz extracted from seven natural samples, one of which is pottery (and so the quartz has been sensitised); these samples contain a range of equivalent doses from $\sim 0.5 \mathrm{~Gy}$ to $\sim 100$ Gy. We have measured single-grain dose distributions on both the EMCCD- and XY-systems using these samples and present average doses, CAM doses (Galbraith et al., 1999), relative standard deviations $(\sigma)$ and fractional grain recovery in Figure 7. We have also made two beta and two gamma dose recovery measurements and these data are also included in Figure 7. In Figure 7a the EMCCD CAM doses are shown against the XY CAM doses. The dashed line has a slope of 1 and passes through the origin; it is clear that the doses measured by the two instruments are indistinguishable. (The inset to Figure 7a shows the unweighted average doses again compared to a line with a slope of 1.) The fraction of grains accepted into the dose distributions is shown in Figure $7 \mathrm{~b}$; for insensitive samples where only a small fraction of the 
grains give useful OSL signals the XY system provides about four times as many accepted grains as the EMCCD. At high recoveries the two systems begin to converge (the XY system detects almost all the grains before the EMCCD). Finally, the inset shows the relative standard deviations given by the two sets of dose distributions, calculated ignoring the individual estimates of uncertainty. Surprisingly, there is no clear tendency for the $X Y$ data set to show less dispersion than the EMCCD data set, despite the fact that the XY data have, on average, uncertainties of about $50 \%$ of those obtained with the EMCCD. We conclude that known sources of uncertainty (in particular Poisson statistics) are not significant compared to other sources of dispersion.

\section{CONCLUSIONS}

We have investigated the use of a recently developed EMCCD-based imaging system to measure quartz single grain OSL and TL signals and have compared our results to those obtained using the standard laserbased single-grain Ris $\varnothing$ attachment in which the luminescence signals are detected using a photomultiplier tube (XY system).

Samples have been measured after loading individual sand-sized grains into the same aluminium sample discs as those used with the $X Y$ system to allow comparison with $X Y$ results, to ensure that individual grains are easily tracked from one measurement to another and to ensure that the inevitable optical cross-talk in any EMMCD-based imaging system is both constrained and reproducible from one measurement to another. We find that the cross-talk using a circular ROI of $450 \mu \mathrm{m}$ in diameter and the measurement geometry of the single grain discs (individual grains are loaded into grain holes, 200-300 $\mu \mathrm{m}$ in diameter, spaced $600 \mu \mathrm{m}$ apart) is $\sim 1 \%$.

We have compared the EMCCD-system with the XY-system and find that the latter is twice as sensitive. When using a PMT as detector Poisson statistics is usually assumed when assigning uncertainties to individual signals. However, when using our EMCCD as detector the recorded signals must be divided by 2 to provide Poisson distributed data; this means that the XY-system is effectively four times as sensitive as the EMCCD-system. Nevertheless, it is possible to use the EMCCD-system to routinely measure natural single-grain quartz distributions. We have measured seven natural samples of different origins and OSL sensitivities using the EMCCD-system and find good agreement with the results from the XY-system both in terms of dose (weighted and unweighted) and variability (relative standard deviation and over-dispersion). Unfortunately, because of the poorer sensitivity the grain recovery for most samples is about four times lower using the EMCCD-system. 
We have investigated the cause of the large variability often observed in quartz single-grain simulation curves using the $\mathrm{XY}$-system and conclude that it is mainly caused by differences in effective stimulation power when the tightly focussed laser light hits the surfaces of different grains. We have also investigated whether there are any differences in the shapes of dose response curves between low power $\left(\mathrm{mW} / \mathrm{cm}^{2}\right.$, green and blue LEDs) and high power (W/ $\mathrm{cm}^{2}$, green laser) stimulation and find that low power stimulation appears to result in DRC saturating at higher doses than those from high power stimulation. Finally, we have investigated whether the unexplained intrinsic variability observed in single-grain measurements could be caused by first SAR cycle sensitivity change by correcting using the TL response to a test dose prior to the measurement of $L_{x}$. We conclude that for this sample (calibration quartz) the variability is increased compared to the standard sensitivity correction using the OSL signal from a subsequent test dose.

We conclude that the EMCCD-system can provide useful measurements of single-grain quartz doses, although at the present level of technology it is about four times less sensitive than the laser/PMT system. 


\section{REFERENCES}

Bailey, R.M., 2003. Paper I: the use of measurement-time dependent single aliquot equivalent-dose estimates from quartz in the identification of incomplete signal resetting. Radiation Measurements 37, 673-683.

Ballarini, M., Wallinga, J., Wintle, A.G., Bos, A.J.J., 2007. A modified SAR protocol for optical dating of individual grains from young quartz samples. Radiation Measurements 42, 360 - 369.

Baril, M., 2004. CCD imaging of the infra-red stimulated luminescence of feldspars. Radiation Measurements 38, 81-86.

Bøtter-Jensen, L., Thomsen, K.J., Jain, M., 2010. Review of optically stimulated luminescence (OSL) instrumental developments for retrospective dosimetry. Radiation Measurements 45, 253-257.

Bøtter-Jensen, L., Andersen, C.E., Duller, G.A.T., Murray, A. S., 2003. Developments in radiation, stimulation and observation facilities in luminescence measurements. Radiation Measurements 37, 535-541.

Choi, J.H., Murray, A.S., Jain, M., Cheong, C.S., Chang, H.W., 2003. Luminescence dating of well-sorted marine terrace sediments on the southeastern coast of Korea. Quaternary Science Reviews 22, 407-421. Clark-Balzan, L., Schwenninger, J-L., 2012. First steps toward spatially resolved OSL dating with electron multiplying charge-coupled devices (EMCCDs): System design and image analysis. Radiation Measurements 47, 797-802.

Duller, G.A.T., 2012a. Cross-talk during single grain optically stimulated luminescence measurements of quartz and feldspar. Radiation Measurements 47, 219-224

Duller, G.A.T., 2012b. Improving the accuracy and precision of equivalent doses determined using the optically stimulated luminescence signal from single grains of quartz. Radiation Measurements 47, 770777.

Duller, G.A.T., 2007. Assessing the error on equivalent dose estimates derived from single aliquot regenerative dose measurements. Ancient TL 25, 15-24.

Duller, G.A.T., Bøtter-Jensen, L., Murray, A.S., Truscott, A.J., 1999. Single grain laser luminescence (SGLL) measurements using a novel automated reader. Nuclear Instruments and Methods in Physics Research B $155,506-514$. 
Duller, G.A.T., Bøtter-Jensen, L., Markey, B.G., 1997. A luminescence imaging system based on a CCD camera. Radiation Measurements 27, 91-99.

Galbraith, R., Roberts, R.G., Laslette, G., Yoshidha, Olley, J., 1999. Optical dating of single and multiple grain Quartz from Jinmium Rock Shelter, Northern Australia. Part I, Experimental design and statistical models. Archaeometry 41, 339-364.

Greilich, S., Wagner, G., 2006. Development of a spatially resolved dating technique using HR-OSL. Radiation Measurements 41, 738-743.

Greilich, S., 2004. Uber die Datierung von Gesteinsoberflächen mittels optisch stimulierter Lumineszenz (The dating of stone surfaces using optically stimulated luminescence). Ph.D. thesis (in German). RuprechtKarls-Universität Heidelberg: Germany.

Greilich, S., Glasmacher, U.A., Wagner, G.A., 2002. Spatially resolved detection of luminescence: a unique tool for archaeochronometry. Naturwissenschaften 89, 371-375.

Hansen, V., Murray, A.S., Buylaert, J-P., Yeo, E-Y., Thomsen, K.J., these proceedings. A new irradiated quartz for beta source calibration. Submitted to Radiation Measurements.

Jain, M., Murray, A.S., Bøtter-Jensen, L., 2003. Characterisation of blue-light stimulated luminescence components in different quartz samples: implications for dose measurement. Radiation Measurements 37, 441-449.

Kook, M.H., Lapp, T., Murray, A.S., Thomsen, K.J., Jain, M., these proceedings. A luminescence imaging system for the routine measurement of single-grain OSL dose distributions. Submitted to Radiation Measurements.

Lapp, T., Kook, M., Murray, A.S, Thomsen, K.J., Buylaert, J.-P., Jain, M., these proceedings. The new luminescence detection and stimulation head for the Ris $\varnothing$ TL/OSL reader. Submitted to Radiation Measurements.

Mundupuzhakal, J., Adhyaru, P., Chauhan, N., Vaghela, H., Shah, M., Chakrabarty, B., Acharya, Y., 2014. FPGA based TL OSL system with EMCCD for luminescence studies. 2014 JINST 9 P04001.

Murray, A.S., Wintle, A.G., 2000. Luminescence dating of quartz using an improved single-aliquot regenerative-dose protocol. Radiation Measurements 32, 57-73. 
Murray, A.S., Roberts, R.G., 1998. Measurement of the equivalent dose in quartz using a regenerative-dose single-aliquot protocol. Radiation Measurements 29, 503-515.

Olko, P., Czopyk, L., Klosowski, M., Waligórski, M.P.R., 2008. Thermoluminescence dosimetry using TLreaders equipped with CCD cameras. Radiation Measurements 43, 864-869.

Richter, D., Richter, A., Dornich, K., 2013. Lexsyg - a new system for luminescence research. Geochronometria 40, 220-228.

Singarayer, J.S., Bailey, R.M., 2004. Component-resolved bleaching spectra of quartz optically stimulated luminescence: preliminary results and implications for dating. Radiation Measurements 38, 111-118.

Singarayer, J.S., Bailey, R.M., 2003. Further investigations of the quartz optically stimulated luminescence components using linear modulation. Radiation Measurements 37, 451-458.

Singhvi, A.K., Stokes, S.C., Chauhan, N., Nagar, Y.C., Jaiswal, M.K., 2011. Changes in natural OSL sensitivity during single aliquots regeneration procedure and their implications for equivalent dose determination. Geochronometria 38 (3), 231-241.

Spooner, N.A., 2000. A photon-counting imaging system (PCIS) for luminescence applications. Radiation Measurements 32, 513-521.

Thomsen, K.J., Murray, A.S., Buylaert, J. P., Jain, M. Submitted to Geochronometria. Application of the Fast Ratio, FR to single grain dose measurement obtained using laser stimulation.

Thomsen, K.J., Murray, A.S., Jain, M., 2012. The dose dependency of the over-dispersion of quartz OSL single grain dose distributions. Radiation Measurements 47, 732-739.

Thomsen, K.J., Bøtter-Jensen, L., Denby, P.M., Moska, P., Murray, A.S., 2006. Developments in luminescence measurement techniques. Radiation Measurements 41, 768-773. 


\section{FIGURE CAPTIONS}

Figure 1: $\quad$ Single grains of calibration (heated and sensitised) quartz measured using the $X Y$ system $(a, c)$ and the EMCCD system (b,d). a) and b) show examples of typical SAR dose response curves (open symbols are recycling points). The insets show the corresponding natural OSL stimulation curve. c) and d) show the resulting dose distributions.

Figure 2: $\quad$ Sensitivity of the EMCCD compared to the PMT on a grain by grain basis. The blue line indicates the linear fit to the data and the dashed line the 1:1 line. The inset shows a close up of the same data. Data points marked in red indicate grain holes significantly affected by cross-talk (see Figure 3 and text for details).

Figure 3: The effect of cross-talk for EMCCD images. a) EMCCD image for a single-grain disc of calibration given a dose of $50 \mathrm{~Gy}$ and preheated to $240{ }^{\circ} \mathrm{C}$ for $10 \mathrm{~s}$. The grain holes marked with red circles are the same as those data points marked in red in Figure 2. b) Blue LED stimulation curve for G82 measured with the EMCCD. The inset shows the same for G81. c) The same as b) but for green-laser stimulation measured with the PMT.

Figure 4: $\quad$ Normalised Blue OSL stimulation curves for calibration quartz for a) six multi-grain aliquots $(3 \mathrm{~mm})$ and b) 93 single grains. The individual multi-grain curves have been fitted using two exponentials and a constant and the average fit is shown in a). The insets show the same data as the main graphs but using a logarithmic y-axis.

Figure 5: $\quad$ Histograms of the characteristic life time (here simplified by using $T_{\frac{1}{2}}$, which indicates the time it takes for the stimulation curve to decrease to $50 \%$ of its initial value) determined for the same single grain using four modes of stimulation. a) green laser stimulation at $10 \%$ power (PMT detection), b) Green laser stimulation at 90\% power (PMT detection), c) Green LED stimulation (EMCCD detection), and d) Blue LED stimulation (EMCCD detection).

Figure 6: $\quad$ a) Single grain calibration quartz correlations between the $110^{\circ} \mathrm{C}$ TL peak and OSL. The TL curves have been summed from 75 to $145^{\circ} \mathrm{C}$ and the OSL summed over the initial $0.5 \mathrm{~s}$ of stimulation. The data has been obtained using the following structure: $\mathrm{TD}_{1}-\mathrm{TL}_{1,180}-$ $\mathrm{PH}_{260,10 \mathrm{~s}}-\mathrm{OSL}_{1}-\mathrm{TD}_{1}-\mathrm{TL}_{2,180}-\mathrm{TD}_{2}-\mathrm{TL}_{220}-\mathrm{OSL}_{2}$, where $\mathrm{TD}_{1}$ is a test dose of $0.5 \mathrm{~Gy}$ and 
$\mathrm{TD}_{2}$ is of $3.5 \mathrm{~Gy}$. Also shown is a linear fit (forced through the origin) to the data. b), c), d) Dose distributions for single grain calibration quartz calibration using different approaches for sensitivity change correction (see legends and text for details).

Figure 7: $\quad$ Summary of the quartz data obtained for natural samples and dose recovery experiments measured using both the EMCCD- and XY-systems. The following natural samples (squares) have been measured: 031301 (Namibia, pottery), 031305 (Namibia, fluvial), 145611 (Ghana, colluvium), 092203 (France, colluvium), H22553 (Russia, fluvial), CSD3 and CSD5 (Portugal, fluvial). Circles represent beta dose recovery experiments (5 Gy CSD3 and 65 Gy 092203). Triangles represent gamma dose recovery experiments (0.5 Gy 031301 and 75 Gy Gy 092203). The dashed line represents the 1:1 line. a) Comparison of CAM doses. The inset shows a comparison of unweighted average doses. b) Comparison of grain recovery, i.e. the number of accepted grains relative to the total number of measured grains. The inset compares the relative standard deviations $\sigma$. 
Figure 1

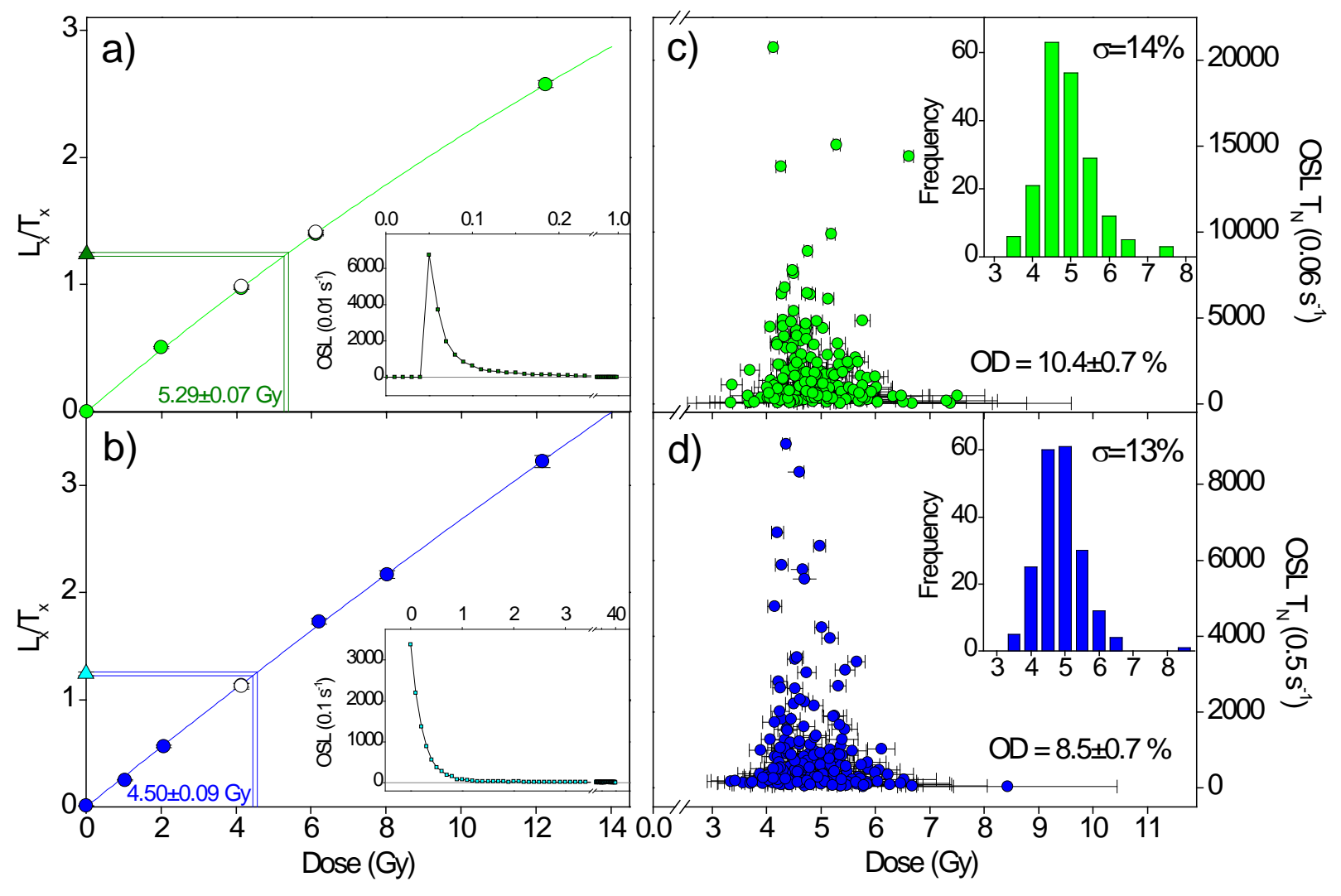


Figure 2

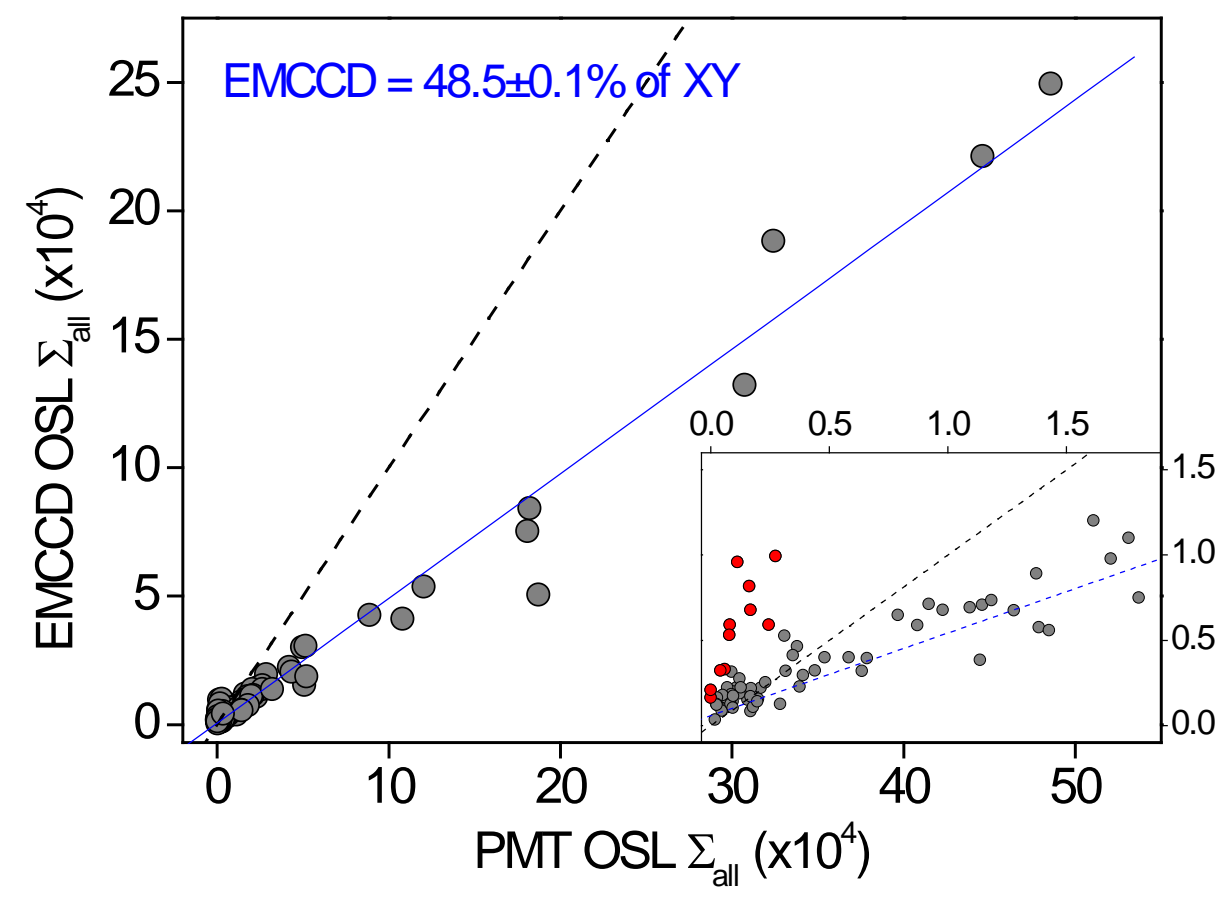


Figure 3
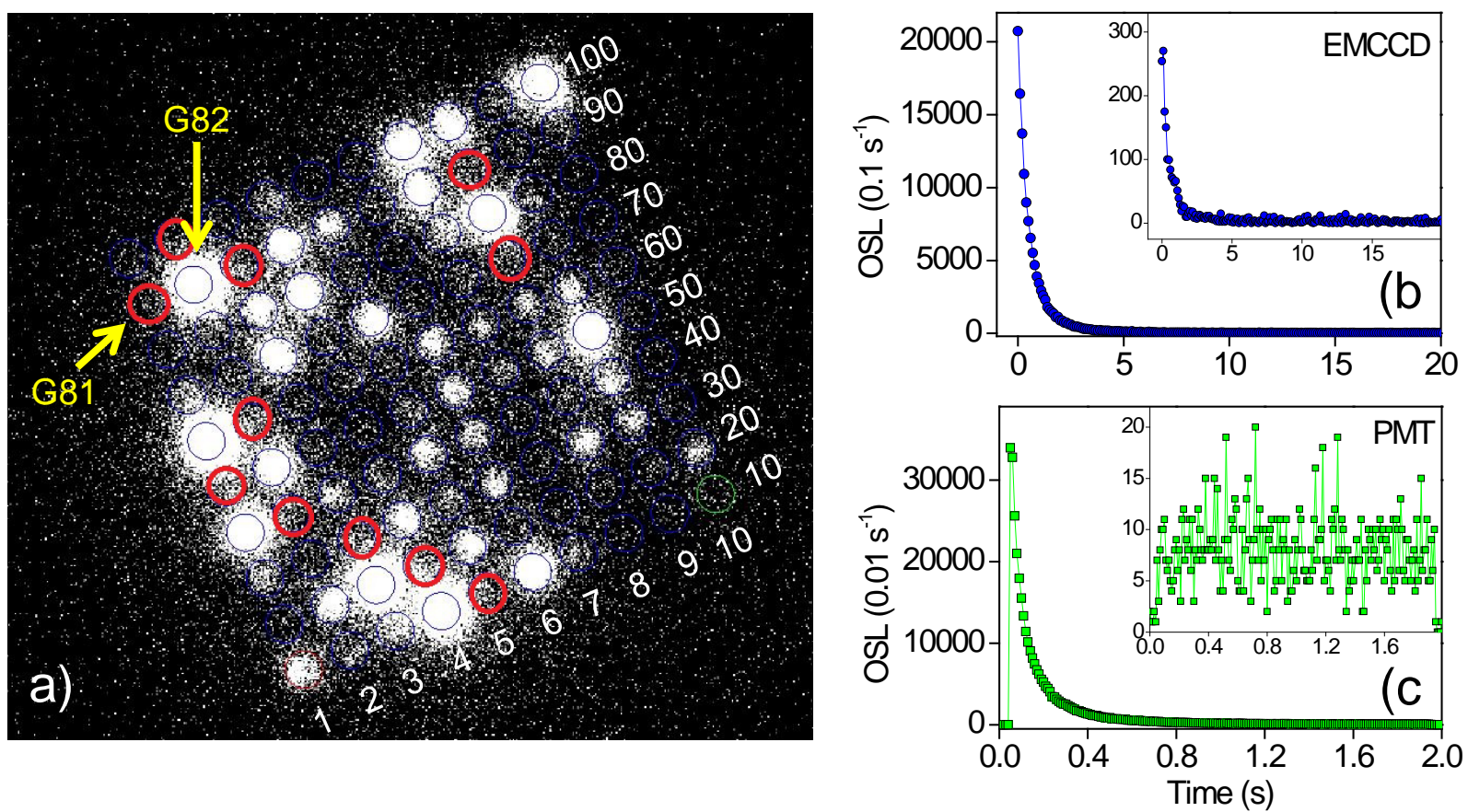
Figure 4
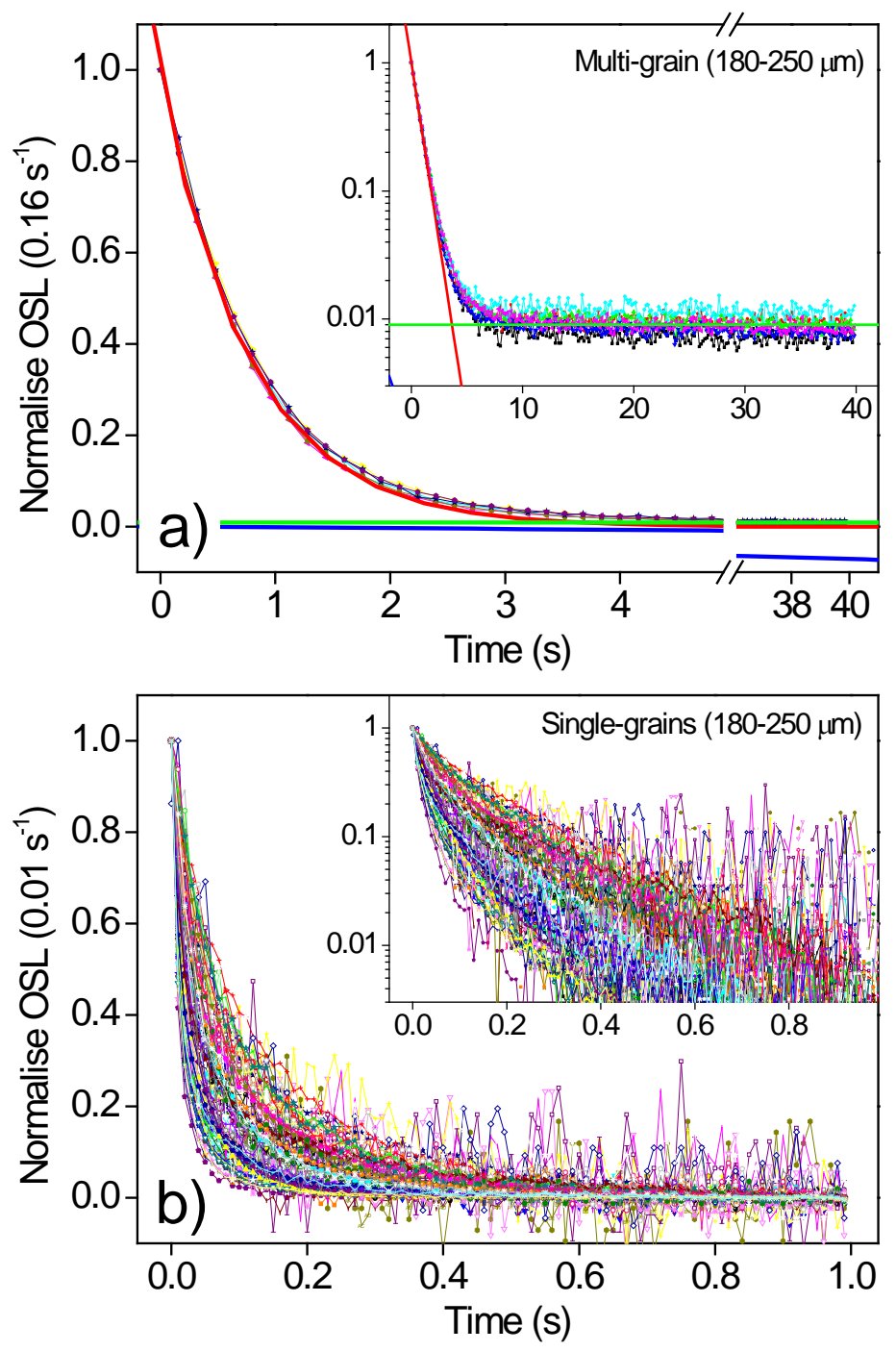
Figure 5

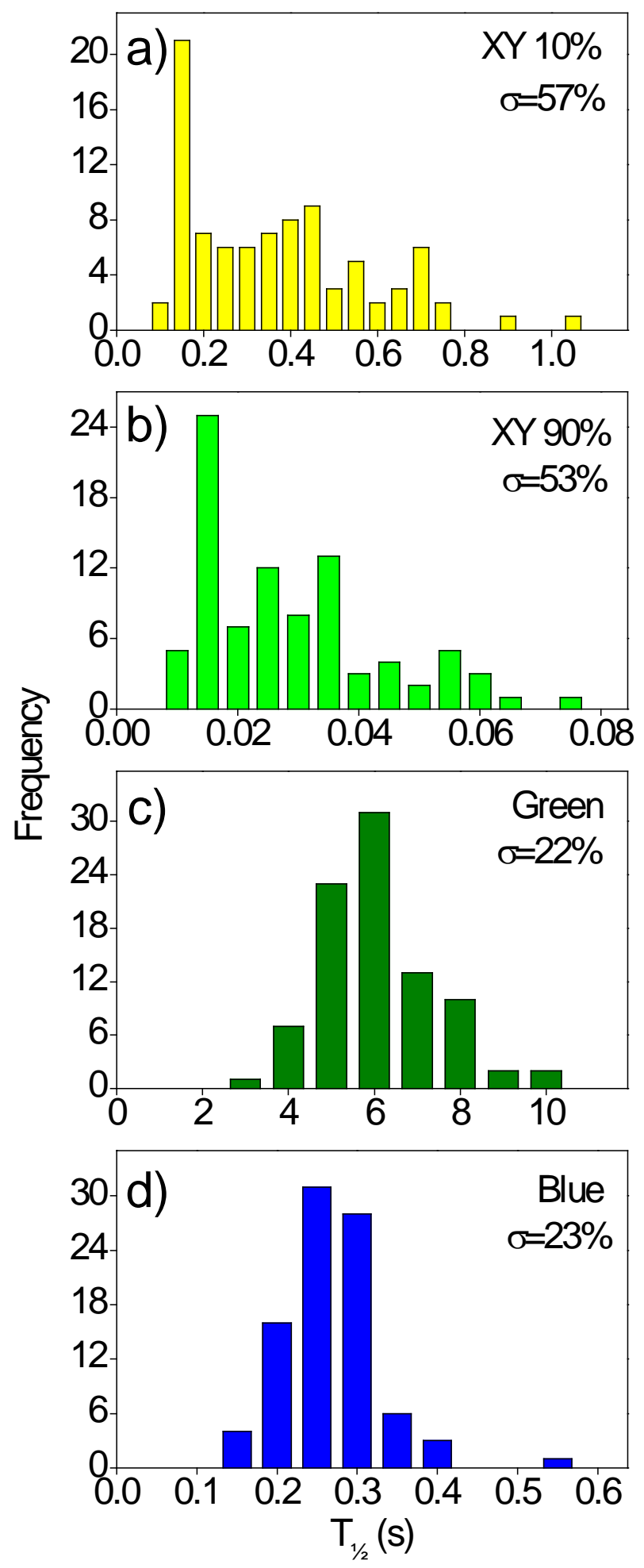


Figure 6
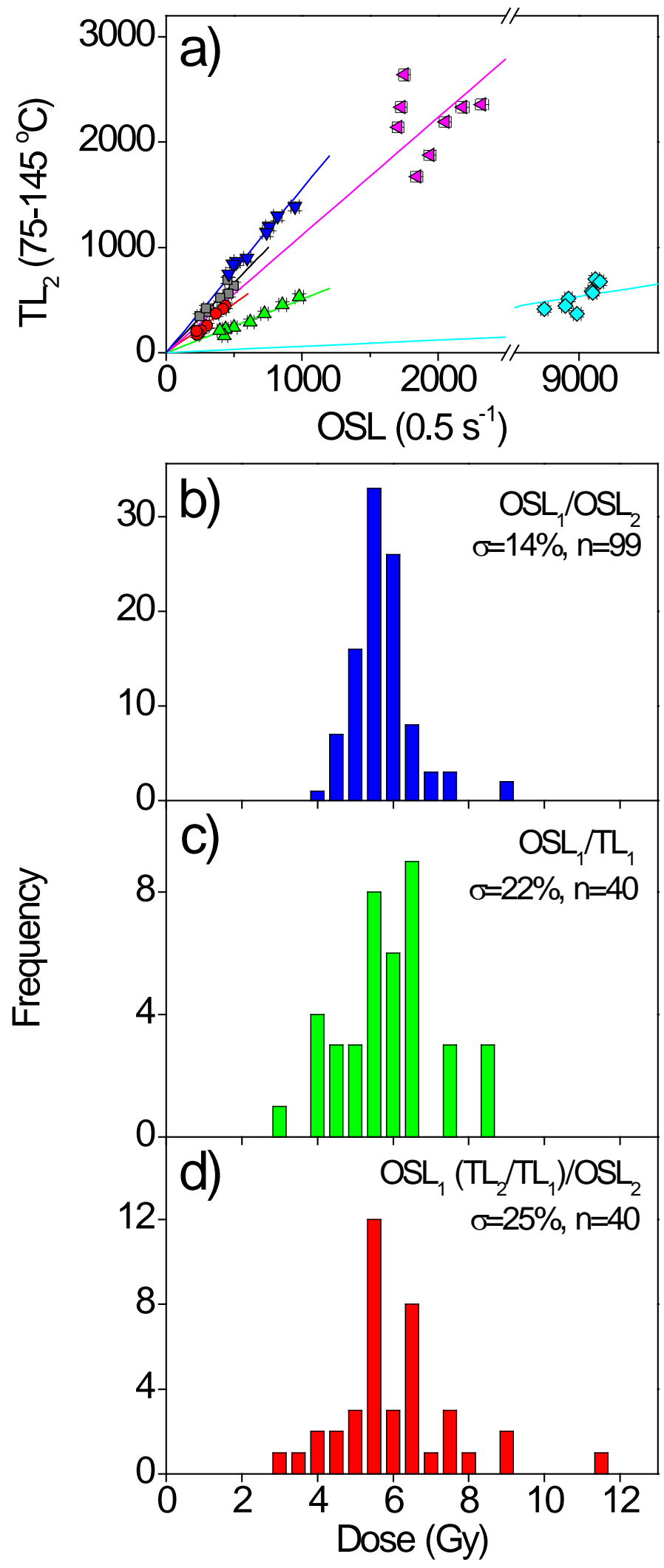
Figure 7
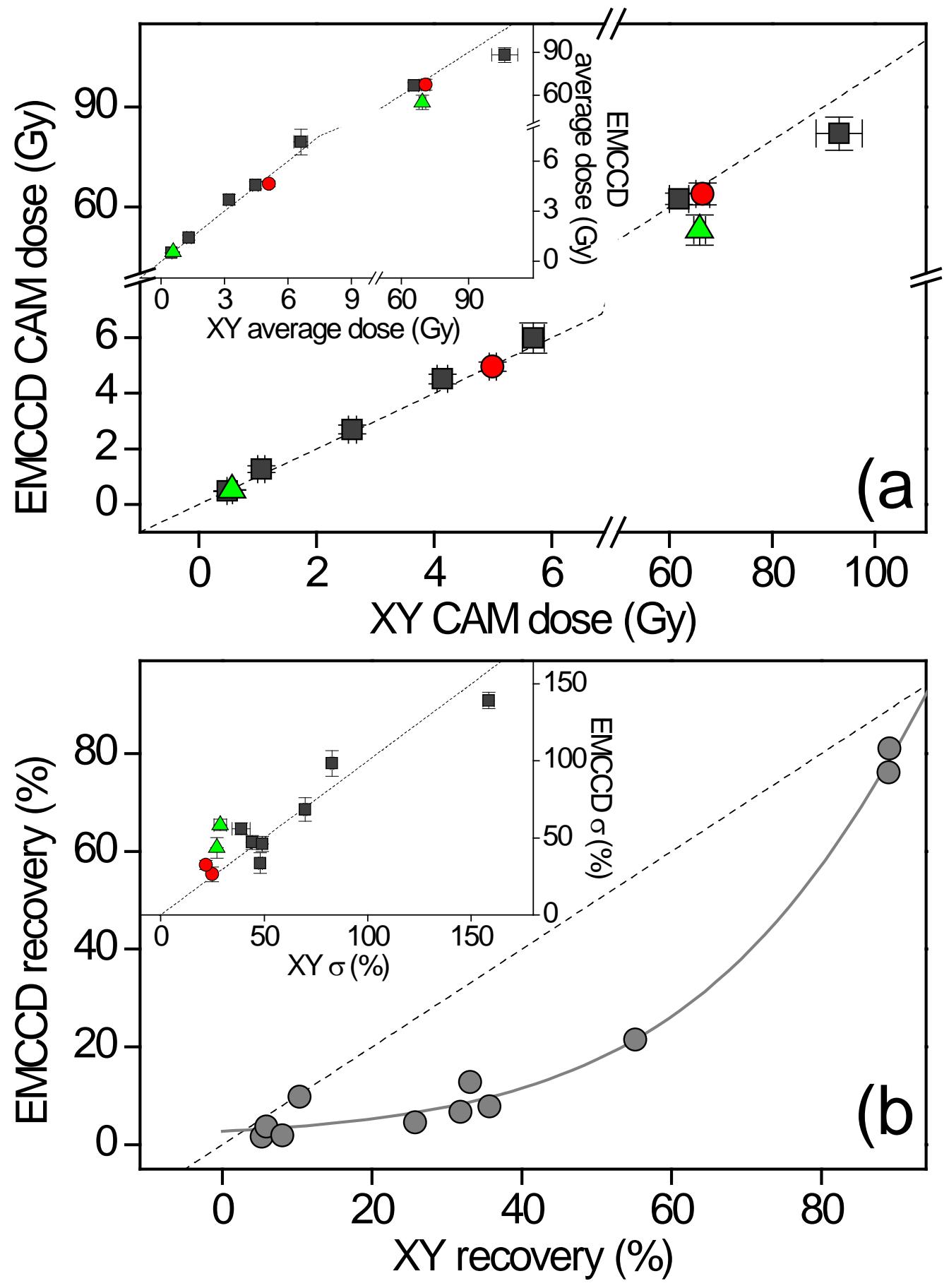